\title{
Randomized Gossiping with Unreliable Communication: Dependent or Independent Node Updates
}

\author{
Guodong Shi, Mikael Johansson and Karl Henrik Johansson
}

\begin{abstract}
This paper studies an asynchronous randomized gossip algorithm under unreliable communication. At each instance, two nodes are selected to meet with a given probability. When nodes meet, two unreliable communication links are established with communication in each direction succeeding with a time-varying probability. It is shown that two particularly interesting cases arise when these communication processes are either perfectly dependent or independent. Necessary and sufficient conditions on the success probability sequence are proposed to ensure almost sure consensus or $\epsilon$-consensus. Weak connectivity is required when the communication is perfectly dependent, while double connectivity is required when the communication is independent. Moreover, it is proven that with odd number of nodes, average preserving turns from almost forever (with probability one for all initial conditions) for perfectly dependent communication, to almost never (with probability zero for almost all initial conditions) for the independent case. This average preserving property does not hold true for general number of nodes. These results indicate the fundamental role the node interactions have in randomized gossip algorithms.
\end{abstract}

Keywords: Gossip algorithms, Unreliable communication, Consensus, Convergence analysis

\section{INTRODUCTION}

The investigation of consensus problems has been widely spread among research communities such as computer science [18], [19], engineering [27], [36], [20], [28] and social science [17], [41], [42]. Deterministic consensus algorithms have been extensively studied for both time-invariant and time-varying communication graphs in the literature, in which efforts were typically devoted to finding proper connectivity conditions which can ensure a desired collective convergence for the considered network [21], [22], [40], [29], [20], [28], [25], [26]. On the other hand, various randomized consensus algorithms have also been studied, motivated by the stochastic nature of information flow over networks [30], [31], [33], [34], [32], [38], [43]. Many sufficient and/or necessary conditions have been established to guarantee a global consensus with probability one [30], [31], [33], [34], [37], [32] under different settings for the randomization of the communication graphs, and convergence rates of randomized consensus algorithms have also been investigated [35], [33], [34].

Gossip algorithms, in which each node communicates with no more than one neighbor in each time slot, were initially motivated by applications in wireless sensor networks and

This work has been supported in part by the Knut and Alice Wallenberg Foundation and the Swedish Research Council. The authors are with ACCESS Linnaeus Centre, School of Electrical Engineering, Royal Institute of Technology, Stockholm 10044, Sweden. Email: guodongs, mikaelj, kallej@kth.se peer-to-peer networks. In [12], averaging problems under gossip communication was studied, averaging times were established and procedures for optimizing algorithm parameters to obtain fast gossiping were given. In [41], a gossip algorithm was used to describe the spread of misinformation on social networks, where the state of each node was viewed as its belief and the randomized gossip algorithm characterized the dynamics of the belief evolution. Researchers have been devoted to studying conditions under which gossip algorithms converge to consensus [9], [10], establishing convergence rates [12], and optimizing the algorithm to reach a faster convergence [11]. However, few works have been looking at the role that unreliable communication between two nodes engaging in gossip play on the overall convergence of the algorithm.

In this paper, we study randomized gossip algorithms under unreliable communication [12], [41]. When two nodes are selected randomly, they establish two unreliable communication links with opposite directions. We suppose the communication on each link succeeds with a time-dependent probability. We propose necessary and sufficient conditions on the success probability sequence to ensure a.s. consensus or $\epsilon$-consensus under perfectly dependent and independent communication, respectively. The fundamental difference with or without symmetry in the node communication is also established, which shows that symmetry missing in the communication may change the overall convergence behavior of the algorithm.

The rest of the paper is organized as follows. In Section II, some preliminary concepts are introduced. We present the network model, the randomized gossip algorithm, and the standing assumptions in Section III. Then main results for perfectly dependent and independent communication are given in Sections IV and V, respectively. Finally, concluding remarks are given in Section VI.

\section{PReliminaries}

In this section, we recall some basic definitions from graph theory [3], stochastic matrices [2] and Bernoulli trials [1].

A directed graph (digraph) $\mathcal{G}=(\mathcal{V}, \mathcal{E})$ consists of a finite set $\mathcal{V}$ of nodes and an arc set $\mathcal{E}$. An element $e=$ $(i, j) \in \mathcal{E}$ is called an arc from node $i \in \mathcal{V}$ and to $j \in \mathcal{V}$. If the arcs are pairwise distinct in an alternating sequence $v_{0} e_{1} v_{1} e_{2} v_{2} \ldots e_{k} v_{k}$ of nodes $v_{i} \in \mathcal{V}$ and arcs $e_{i}=\left(v_{i-1}, v_{i}\right) \in \mathcal{E}$ for $i=1,2, \ldots, k$, the sequence is called a (directed) path with length $k$. A path with no repeated nodes is called a simple path. A path from $i$ to $j$ is denoted as $i \rightarrow j$, and the length of $i \rightarrow j$ is denoted as $|i \rightarrow j|$. 
If there exists a path from node $i$ to node $j$, then node $j$ is said to be reachable from node $i$. Each node is thought to be reachable by itself. A node $v$ from which any other node is reachable is called a center (or a root) of $\mathcal{G}$. A digraph $\mathcal{G}$ is said to be strongly connected if it contains path $i \rightarrow j$ and $j \rightarrow i$ for every pair of nodes $i$ and $j$, and quasi-strongly connected if $\mathcal{G}$ has a center [8].

The converse graph, $\mathcal{G}^{T}$ of digraph $\mathcal{G}=(\mathcal{V}, \mathcal{E})$, is defined as the graph obtained by reversing the orientation of all arcs in $\mathcal{E}$. The distance from $i$ to $j$ in a digraph $\mathcal{G}, d(i, j)$, is the length of a shortest simple path $i \rightarrow j$ if $j$ is reachable from $i$, and the diameter of $\mathcal{G}$ is $\operatorname{Diam}(\mathcal{G})=\max \{d(i, j) \mid i, j \in$ $\mathcal{V}, j$ is reachable from $i\}$.

The union of the two digraphs with the same node set $\mathcal{G}_{1}=\left(\mathcal{V}, \mathcal{E}_{1}\right)$ and $\mathcal{G}_{2}=\left(\mathcal{V}, \mathcal{E}_{2}\right)$ is defined as $\mathcal{G}_{1} \cup \mathcal{G}_{2}=$ $\left(\mathcal{V}, \mathcal{E}_{1} \cup \mathcal{E}_{2}\right)$; we denote $\mathcal{G}_{1} \subseteq \mathcal{G}_{2}$ if $\mathcal{E}_{1} \subseteq \mathcal{E}_{2}$.

A digraph $\mathcal{G}$ is said to be bidirectional if for every two nodes $i$ and $j,(i, j) \in \mathcal{E}$ if and only if $(j, i) \in \mathcal{E}$. A bidirectional graph $\mathcal{G}$ is said to be connected if there is a path between any two nodes. A digraph $\mathcal{G}$ is said to be weakly connected if it is connected as a bidirectional graph when all the arc directions are ignored. Strongly or quasi-strongly connected digraphs are hence always weakly connected.

A finite square matrix $M=\left[m_{i j}\right] \in \mathbb{R}^{n \times n}$ is called stochastic if $m_{i j} \geq 0$ for all $i, j$ and $\sum_{j} m_{i j}=1$ for all $i$. For a stochastic matrix $M$, introduce

$$
\delta(M)=\max _{j} \max _{\alpha, \beta}\left|m_{\alpha j}-m_{\beta j}\right|
$$

and

$$
\lambda(M)=1-\min _{\alpha, \beta} \sum_{j} \min \left\{m_{\alpha j}, m_{\beta j}\right\} .
$$

If $\lambda(M)<1$ we call $M$ a scrambling matrix. The following lemma can be found in [16].

Lemma 2.1: For any $k \geq 1$ stochastic matrices $M_{1}, \ldots, M_{k}$,

$$
\delta\left(M_{1} M_{2} \ldots M_{k}\right) \leq \prod_{i=1}^{k} \lambda\left(M_{i}\right) .
$$

A stochastic matrix $M=\left[m_{i j}\right] \stackrel{i=1}{\in} \mathbb{R}^{n \times n}$ is called doubly stochastic if also $M^{T}$ is stochastic.

For any matrix $P=\left[p_{i j}\right] \in \mathbb{R}^{n \times n}$ with nonnegative entries, we can associate a unique digraph $\mathcal{G}_{P}=\left\{\mathcal{V}, \mathcal{E}_{P}\right\}$ with node set $\mathcal{V}=\{1, \ldots, n\}$ such that $(j, i) \in \mathcal{E}_{P}$ if and only if $p_{i j}>0$. We call $\mathcal{G}_{P}$ the induced graph of $P$.

A sequence of independently distributed Bernoulli trials is a finite or infinite sequence of independent random variables $\mathfrak{B}_{0}, \mathfrak{B}_{1}, \mathfrak{B}_{2}, \ldots$, such that

(i) For each $k \geq 0, \mathfrak{B}_{k}$ equals either 0 or 1 ;

(ii) For each $k$, the probability that $\mathfrak{B}_{k}=1$ is $p_{k}$.

We call $p_{k}$ the success probability for time $k$. The sequence of integers

$$
0 \leq \zeta_{1}<\zeta_{2}<\cdots: \quad \mathfrak{B}_{\zeta_{m}}=1
$$

is the Bernoulli (success) sequence associated with the sequence of Bernoulli trials with $\zeta_{m}$ marking the time of the $m$ 'th success.

\section{PRoblem Definition}

In this section, we present the considered network model and define the problem of interest.

\section{A. Node Pair Selection Process}

Consider a network with node set $\mathcal{V}=\{1, \ldots, n\}(n \geq$ $3)$. Let the digraph $\mathcal{G}_{0}=\left(\mathcal{V}, \mathcal{E}_{0}\right)$ denote the underlying graph of the considered network. The underlying graph indicates potential interactions between nodes. We use the asynchronous time model introduced in [12] to describe node interactions. Each node meets other nodes at independent time instances defined by a rate-one Poisson process. This is to say, the inter-meeting times at each node follows a rateone exponential distribution. Without loss of generality, we can assume that at most one node is active at one given instance. Let $x_{i}(k) \in \mathbb{R}$ denote the state (value) of node $i$ at the $k$ 'th meeting slot among all the nodes.

Node interactions are characterized by an $n \times n$ matrix $A=\left[a_{i j}\right]$, where $a_{i j} \geq 0$ for all $i, j=1, \ldots, n$ and $a_{i j}>0$ if and only if $(j, i) \in \mathcal{E}_{0}$. We assume $A$ is a stochastic matrix. The node pair selection process for the gossip algorithm is defined as follows.

Definition 3.1: (Node Pair Selection Process) At each time $k \geq 0$,

(i) A node $i \in \mathcal{V}$ is drawn with probability $1 / n$;

(ii) Node $i$ picks the pair $(i, j)$ with probability $a_{i j}$.

Note that, by the definition of the node pair selection process, the underlying graph $\mathcal{G}_{0}$ is actually the same as $\mathcal{G}_{A}$, the induced graph of the meeting probability matrix $A$. For $\mathcal{G}_{0}$, we use the following assumption.

A1. (Weak Connectivity) The underlying graph $\mathcal{G}_{0}$ is weakly connected.

Remark 3.1: In order to guarantee convergence for the gossip algorithm discussed below, A1 cannot be further weakened since the network is essentially divided into two isolated parts, and a convergence for the whole network is thus impossible.

\section{B. Node Communication Process}

When pair $(i, j)$ is selected, both nodes try to set their states equal to the average of their current states. To this end, two communication links with opposite directions are established between the two nodes.

The communication process is defined as follows.

Definition 3.2: (Node Communication Process) Independent with time and the node pair selection process,

(i) $\mathbf{P}\left(\mathbb{E}_{i j}^{+}(k)\right)=P_{k}^{+}$with $0 \leq P_{k}^{+} \leq 1$, where event $\mathbb{E}_{i j}^{+}(k)=$ nnode $i$ receives $x_{j}(k)$ when $(i, j)$ is selected at time $k\}$;

(ii) $\mathbf{P}\left(\mathbb{E}_{i j}^{-}(k)\right)=P_{k}^{-}$with $0 \leq P_{k}^{-} \leq 1$, where event $\mathbb{E}_{i j}^{-}(k)=$ nnode $j$ receives $x_{i}(k)$ when $(i, j)$ is selected at time $k\}$.

If a node fails to receive the value of the other node, it will keep its current state. Note that we do not impose the independence between $i$ receiving $x_{j}(k)$ and $j$ receiving $x_{i}(k)$ when pair $(i, j)$ is selected. In fact, we will study how 
such potential dependence in the communication process influence the convergence of the gossip algorithm.

Remark 3.2: A randomized gossip algorithm can also be viewed as belief propagation in a social network, where $x_{i}(k)$ represents the belief of node $i$. Then the communication process naturally captures the loss of 'trust' when two nodes meet and exchange opinions [41], [42]. Therefore, from a social network viewpoint, the discussion in this paper on the convergence property of the gossip algorithm establishes the influence of missing 'trust' in belief agreement.

\section{Problem}

Let the initial condition be $x^{0}=x\left(k_{0}\right)=$ $\left(x_{1}\left(k_{0}\right) \ldots x_{n}\left(k_{0}\right)\right)^{T} \in \mathbb{R}^{n}$, where $k_{0} \geq 0$ is an arbitrary integer. According to the node pair selection process and the communication process, the iteration of the gossip algorithm can be expressed as:

$$
x_{i}(k+1)= \begin{cases}\frac{1}{2} x_{i}(k)+\frac{1}{2} x_{j}(k), & \text { if } \mathcal{M}^{\langle i, j\rangle} \text { happens } \\ x_{i}(k), & \text { otherwise }\end{cases}
$$

where $\mathcal{M}_{k}^{\langle i, j\rangle} \doteq\{$ pair $(i, j)$ is selected or pair $(j, i)$ is selected, and $i$ receives $x_{j}(k)$ at time $k$ \} denotes the event that node $i$ successfully updates at time $k$. According to the definitions above, we have

$$
\mathbf{P}\left(\mathcal{M}_{k}^{\langle i, j\rangle}\right)=\frac{a_{i j}}{n} P_{k}^{+}+\frac{a_{j i}}{n} P_{k}^{-}
$$

and

$$
\mathbf{P}\left(\mathcal{M}_{k}^{\langle j, i\rangle}\right)=\frac{a_{j i}}{n} P_{k}^{+}+\frac{a_{i j}}{n} P_{k}^{-} .
$$

Therefore, the two events, $\mathcal{M}_{k}^{\langle i, j\rangle}$ and $\mathcal{M}_{k}^{\langle j, i\rangle}$, are not necessarily symmetric in their probabilities, due to the potential asymmetry of the meeting matrix $A$. Moreover, the events are not as follows from the structure of the node pair selection process (even if $A$ is symmetric).

In this paper, we study the convergence of the randomized gossip consensus algorithm and the time it takes for the network to reach a consensus. Let $x\left(k ; k_{0}, x^{0}\right)=$ $\left(x_{1}\left(k ; k_{0}, x_{1}\left(k_{0}\right)\right) \ldots x_{n}\left(k ; k_{0}, x_{n}\left(k_{0}\right)\right)\right)^{T} \in \mathbb{R}^{n}$ be the random process driven by the randomized algorithm (5). When it is clear from the context, we will identify $x\left(k ; k_{0}, x^{0}\right)$ with $x(k)$.

Denote

$$
H(k) \doteq \max _{i=1, \ldots, n} x_{i}(k), \quad h(k) \doteq \min _{i=1, \ldots, n} x_{i}(k)
$$

as the maximum and minimum states among all nodes, respectively, and define $\mathcal{H}(k) \doteq H(k)-h(k)$ as the consensus metric. We introduce the following definition.

Definition 3.3: (i) A global a.s. consensus is achieved if

$$
\mathbf{P}\left(\lim _{k \rightarrow \infty} \mathcal{H}(k)=0\right)=1
$$

for any initial condition $x^{0} \in \mathbb{R}^{n}$.

(ii) Let the $\epsilon$-computation time be

$T_{\text {com }}(\epsilon) \doteq \sup _{x^{0} \in \mathbb{R}^{n}, k_{0} \geq 0} \inf \left\{k-k_{0}: \mathbf{P}\left(\frac{\mathcal{H}(k)}{\mathcal{H}\left(k_{0}\right)} \geq \epsilon\right) \leq \epsilon\right\}$
Then a global a.s. $\epsilon$-consensus is achieved if

$$
T_{\text {com }}(\epsilon)=O\left(\log \epsilon^{-1}\right)
$$

where by definition $f(\epsilon)=O(g(\epsilon))$ means that $\limsup _{\epsilon \rightarrow 0} f(\epsilon) / g(\epsilon)<\infty$ is a nonzero constant.

\section{Perfectly Dependent Communication}

In this section, we study the case when the communication between nodes $i$ and $j$ is perfectly dependent, as described in the following assumption.

A2. (Perfectly Dependent Communication) The communication events $\mathbb{E}_{i j}^{+}(k)=\mathbb{E}_{i j}^{-}(k)$ except for a set with probability zero for all $k$.

Note that, A2 is equivalent to assuming that $\mathbf{P}\left(\mathbb{E}_{i j}^{+}(k) \mid \mathbb{E}_{i j}^{-}(k)\right)=\mathbf{P}\left(\mathbb{E}_{i j}^{-}(k) \mid \mathbb{E}_{i j}^{+}(k)\right)=1$. Hence, we have $P_{k}^{+}=P_{k}^{-}$and at each time instant, with probability $P_{k} \doteq P_{k}^{+}=P_{k}^{-}$both $\mathbb{E}_{i j}^{+}(k)$ and $\mathbb{E}_{i j}^{-}(k)$ occur, and with probability $1-P_{k}$ they both fail. With A2, the gossip algorithm can be expressed as

$$
x(k+1)=W(k) x(k),
$$

where $W(k)$ is the random matrix satisfying

$$
W(k)=W_{\langle i j\rangle} \doteq I-\frac{\left(e_{i}-e_{j}\right)\left(e_{i}-e_{j}\right)^{T}}{2}
$$

with probability $\frac{a_{i j}+a_{j i}}{n} P_{k}$ for $i \neq j$, with $e_{m}=$ $\left(\begin{array}{lllll}0 & \ldots & 1 & 0 \ldots 0\end{array}\right)^{T}$ denoting the $n \times 1$ unit vector whose $m$ 'th component is 1 . Moreover, $\mathbf{P}\left(W(k)=W_{\langle i i\rangle}=I\right)=$ $1-\sum_{i>j} \frac{a_{i j}+a_{j i}}{n} P_{k}$.

Denote $D=\operatorname{diag}\left(d_{1} \ldots d_{n}\right)$ with $d_{i}=\sum_{j=1}^{n}\left(a_{i j}+a_{j i}\right)$. The main result on a.s. consensus and $\epsilon$-consensus for the considered gossip algorithm under perfectly dependent communication is stated as follows.

Theorem 4.1: Suppose A1 (Weak Connectivity) and A2 (Perfectly Dependent Communication) hold. A global a.s. consensus is achieved if and only if $\sum_{k=0}^{\infty} P_{k}=\infty$.

Theorem 4.2: Suppose A1 (Weak Connectivity) and A2 (Perfectly Dependent Communication) hold. A global a.s. $\epsilon$ consensus is achieved if and only if there exist a constant $p_{*}>0$ and an integer $T_{*} \geq 1$ such that $\sum_{k=m}^{m+T_{*}-1} P_{k} \geq p_{*}$ for all $m \geq 0$. In fact, we have

$$
T_{\mathrm{com}}(\epsilon) \leq 3\left[\log \left(1-\frac{\lambda_{2}^{*} p_{*}}{2 n T_{*}}\right)^{-1}\right]^{-1} \log \epsilon^{-1}+O(1)
$$

where $\lambda_{2}^{*}$ is the second smallest eigenvalue of $D-\left(A+A^{T}\right)$.

Let the random variable $\xi\left(k_{0}, x^{0}\right)$ denote the consensus limit (supposed to exist), i.e.,

$$
\lim _{k \rightarrow \infty} x_{i}(k)=\xi, \quad \text { a.s. } \quad i=1, \ldots, n .
$$

Denote $x_{\text {ave }}=\sum_{i=1}^{n} x_{i}\left(k_{0}\right) / n$ be the average of the initial values among the network. Then the following conclusion holds showing that average is preserved almost surely with perfectly dependent communication. 
Theorem 4.3: Suppose A1 (Weak Connectivity) and A2 (Perfectly Dependent Communication) hold. Then for all initial conditions $x^{0}=x\left(k_{0}\right) \in \mathbb{R}^{n}$, we have

$$
\mathbf{P}\left(\sum_{i=1}^{n} x_{i}(k)=n x_{\text {ave }}, \quad k \geq k_{0}\right)=1 .
$$

Consequently, we have $\mathbf{P}\left(\xi=x_{\text {ave }}\right)=1$ if the consensus limit exists.

In the following two subsections, we will present the proof of Theorems 4.1 and 4.2, respectively. Theorem 4.3 follows trivially from the proof Theorem 4.1 .

The upcoming analysis relies on the following well-known lemmas.

Lemma 4.1: Suppose $0 \leq b_{k}<1$ for all $k$. Then $\sum_{k=0}^{\infty} b_{k}=\infty$ if and only if $\prod_{k=0}^{\infty}\left(1-b_{k}\right)=0$.

Lemma 4.2: $\log (1-t) \geq-2 t$ for all $0 \leq t \leq 1 / 2$.

\section{A. Proof of Theorem 4.1}

(Sufficiency.) This part of analysis is based on a similar argument as is used in [12]. Define $L(k)=\sum_{i=1}^{n} \mid x_{i}(k)-$ $\left.x_{\text {ave }}\right|^{2}$, where $|\cdot|$ represents the Euclidean norm of a vector or the absolute value of a scalar.

It is easy to verify that for every possible sample and fixed instance $k, W_{\langle i j\rangle}$ of the random matrix $W(k)$ defined in (8) and (9), we have

(i). $W_{\langle i j\rangle}$ is a doubly stochastic matrix, i.e., $W_{\langle i j\rangle} \mathbf{1}=\mathbf{1}$ and $\mathbf{1}^{T} W_{\langle i j\rangle}=\mathbf{1}^{T}$;

(ii). $W_{\langle i j\rangle}$ is a projection matrix, i.e., $W_{\langle i j\rangle}=W_{\langle i j\rangle}^{T} W_{\langle i j\rangle}$.

Based on a similar analysis used in [12], it is not hard to obtain

$$
\mathbf{E}(L(k+1)) \leq \prod_{i=k_{0}}^{k}\left(1-\frac{\lambda_{2}^{*}}{2 n} P_{i}\right) L\left(k_{0}\right)
$$

where $\lambda_{2}^{*}$ is the second smallest eigenvalue of $D-\left(A+A^{T}\right)$. Therefore, based on Lemma 4.1 and Fatou's lemma, we have

$$
\mathbf{E}\left(\lim _{k \rightarrow \infty} L(k)\right) \leq \lim _{k \rightarrow \infty} \mathbf{E}(L(k))=0,
$$

where $\lim _{k \rightarrow \infty} L(k)$ exits simply from the fact that it is nonincreasing. This immediately implies

$$
\mathbf{P}\left(\lim _{k \rightarrow \infty} x_{i}(k)=x_{\text {ave }}\right)=1 .
$$

The sufficiency claim of the theorem thus holds.

(Necessity.) From the definition of the gossip algorithm, we have

$$
\begin{aligned}
\mathbf{P}\left(x_{i}(k+1)=x_{i}(k)\right) & \geq 1-P_{k} \sum_{j=1, j \neq i}^{n} \frac{1}{n}\left(a_{i j}+a_{j i}\right) \\
& \doteq 1-h_{i} P_{k},
\end{aligned}
$$

where $h_{i}=\sum_{j=1, j \neq i} \frac{1}{n}\left(a_{i j}+a_{j i}\right), i=1, \ldots, n$. Noting the fact that

$$
\sum_{i=1}^{n} h_{i}=\sum_{i=1}^{n} \sum_{j=1, j \neq i}^{n} \frac{1}{n}\left(a_{i j}+a_{j i}\right)=2-\sum_{i=1}^{n} a_{i i} \leq 2,
$$

there exists at least one node $\alpha_{1} \in \mathcal{V}$ such that $h_{\alpha_{1}}<1$ since $n \geq 3$. Moreover, assumption A1 further guarantees that all $h_{i}>0, i=1, \ldots, n$, which implies that there exists another node $\alpha_{2} \in \mathcal{V}$ such that $h_{\alpha_{2}}<1$.

Therefore, based on Lemma 4.1,

$$
\mathbf{P}\left(x_{\alpha_{i}}(k)=x_{\alpha_{i}}\left(k_{0}\right), k \geq k_{0}\right) \geq \prod_{k=k_{0}}^{\infty}\left(1-h_{\alpha_{i}} P_{k}\right)>0
$$

for $i=1,2$. Consequently, choosing $x_{\alpha_{1}}\left(k_{0}\right) \neq x_{\alpha_{2}}\left(k_{0}\right)$, consensus will fail with probability $\sigma_{1} \sigma_{2}>0$. This completes the proof.

\section{B. Proof of Theorem 4.2}

The sufficiency proof is based on (13) and Markov's inequality. The necessity statement holds by a contradiction argument investigating $\alpha_{1}, \alpha_{2}$ as in the proof of Theorem 4.1. We refer to [44] for details.

\section{INDEPENDENT COMMUNICATION}

In this section, we focus on the case when the communication between nodes $i$ and $j$ is independent, as described in the following assumption.

A3. (Independent Communication) The communication events $\mathbb{E}_{i j}^{+}(k)$ and $\mathbb{E}_{i j}^{-}(k)$ are independent for all $k$.

In order to establish the convergence results under independent communication, beyond assumption A1, we need the following condition for the underlying connectivity.

A4. (Double Connectivity) Both the underlying graph $\mathcal{G}_{0}$ and its converse graph $\mathcal{G}_{0}^{T}$ are quasi-strongly connected.

Remark 5.1: Note that the condition of $\mathcal{G}_{0}$ being strongly connected implies A4, but not vice versa. Moreover, it is not hard to see that $\mathcal{G}_{0}=\mathcal{G}_{A}$, and $\mathcal{G}_{0}^{T}=\mathcal{G}_{A^{T}}$, where $\mathcal{G}_{A}$ and $\mathcal{G}_{A^{T}}$ are the induced graph of $A$ and $A^{T}$, respectively.

We now present the main results on the convergence of the gossip algorithm under independent communication as follows.

Theorem 5.1: Suppose A3 (Independent Communication) and A4 (Double Connectivity) hold. A global a.s. consensus is achieved if and only if $\sum_{k=0}^{\infty}\left(P_{k}^{+}+P_{k}^{-}\right)=\infty$.

Denote $d_{*}=\max \left\{\operatorname{diam}\left(\mathcal{G}_{\mathrm{A}}\right), \operatorname{diam}\left(\mathcal{G}_{\mathrm{A}^{\mathrm{T}}}\right)\right\}$, where $\operatorname{diam}\left(\mathcal{G}_{\mathrm{A}}\right)$ and $\operatorname{diam}\left(\mathcal{G}_{\mathrm{A}^{\mathrm{T}}}\right)$ represent the diameter of the induced graph of $A$ and $A^{T}$, respectively. Take $E_{*}=$ $\left|\mathcal{E}_{0}\right|-\sum_{i=1}^{n} \operatorname{sgn}\left(a_{i i}\right)$, where $\left|\mathcal{E}_{0}\right|$ represents the number of elements in $\mathcal{E}_{0}$, and $\operatorname{sgn}(z)$ is the sign function. Introduce $a_{*}=\min \left\{a_{i j}: a_{i j}>0, i, j=1, \ldots, n, i \neq j\right\}$ as the lower bound of the nonzero and non-diagonal entries in the meeting probability matrix $A$.

Theorem 5.2: Suppose A3 (Independent Communication) and A4 (Double Connectivity) hold. A global a.s. $\epsilon$ consensus is achieved if and only if there exist a constant $p_{*}>0$ and an integer $T_{*} \geq 1$ such that $\sum_{k=m}^{s+T_{*}-1}\left(P_{k}^{+}+\right.$ $\left.P_{k}^{-}\right) \geq p_{*}$ for all $m \geq 0$. In this case, we have

$$
T_{\text {com }}(\epsilon) \leq \frac{4 T_{*} \theta_{0} / p_{*}}{\log \left(1-\left(\frac{a_{*}}{4 n}\right)^{\theta_{0}}\right)^{-1}} \log \epsilon^{-1}+O(1),
$$

where $\theta_{0} \doteq\left(2 d_{*}-1\right)\left(2 E_{*}-1\right)$. 
Theorem 5.3: Suppose A3 (Independent Communication) holds and the consensus limit exists. Then $\mathbf{E}(\xi)=x_{\text {ave }}$ if $P_{k}^{+}=P_{k}^{-}$for all $k$.

Theorem 5.3 holds trivially since $\mathbf{E}(W(k))$ is a doubly stochastic matrix for all $k$. Furthermore, we have another conclusion showing that whenever the consensus limit $\xi$ exists, the average can almost never be preserved.

Theorem 5.4: Suppose A3 (Independent Communication) holds and the number of nodes, $n$, is odd. Assume that $P_{k}^{+}, P_{k}^{-} \in[0,1-\varepsilon]$ for all $k \geq 0$ with $\varepsilon$ an arbitrary positive number. Then for any $k_{0} \geq 0$ and for almost all initial conditions $x^{0}=x\left(k_{0}\right) \in \mathbb{R}^{n}$, we have

$$
\mathbf{P}\left(\sum_{i=1}^{n} x_{i}(k)=n x_{\text {ave }}, \quad k \geq k_{0}\right)=0
$$

if the consensus limit $\xi$ exists.

For the non-conservativeness of A4 (Double Connectivity) to ensure a consensus under independent communication, we have the following conclusion, which follows from a similar argument as Remark 3.1.

Proposition 5.1: Suppose A3 (Independent Communication) holds. Then the condition $\sum_{k=0}^{\infty}\left(P_{k}^{+}+P_{k}^{-}\right)=\infty$ always implies an a.s. consensus only if A4 (Double Connectivity) holds.

\section{A. Bernoulli Communication Links}

Define two (independent) sequences of independent Bernoulli trials

$$
\begin{aligned}
& \mathfrak{B}_{0}^{+}, \mathfrak{B}_{1}^{+}, \mathfrak{B}_{2}^{+}, \ldots, \\
& \mathfrak{B}_{0}^{-}, \mathfrak{B}_{1}^{-}, \mathfrak{B}_{2}^{-}, \ldots,
\end{aligned}
$$

such that $\mathbf{P}\left(\mathfrak{B}_{k}^{+}=1\right)=P_{k}^{+}$and $\mathbf{P}\left(\mathfrak{B}_{k}^{-}=1\right)=P_{k}^{-}$. Then let

$$
\mathfrak{B}_{0}, \mathfrak{B}_{1}, \mathfrak{B}_{2}, \ldots,
$$

denote the independent Bernoulli trials given by $\mathfrak{B}_{k}=1$ if and only if $\mathfrak{B}_{k}^{-}+\mathfrak{B}_{k}^{+} \geq 1$.

The following lemma holds on the success times of $\left\{\mathfrak{B}_{k}\right\}_{0}^{\infty}$.

Lemma 5.1: $\mathbf{P}\left(\right.$ for all $k_{0} \geq 0, \quad \mathfrak{B}_{k}=$ 1 for infinitely many $\left.k \geq k_{0}\right)=1$ if and only if $\sum_{k=0}^{\infty}\left(P_{k}^{+}+P_{k}^{-}\right)=\infty$.

\section{B. Products of Transition Matrices}

The considered gossip algorithm is determined by the possible samples of the transition matrix $W(k)$. Denote $\mathbb{M}=\mathbb{M}_{1} \cup \mathbb{M}_{2}$ with

$$
\mathbb{M}_{1}=\left\{I-\frac{e_{i}\left(e_{i}-e_{j}\right)^{T}}{2}: \quad a_{i j}+a_{j i}>0, i \neq j\right\}
$$

and

$$
\mathbb{M}_{2}=\left\{I-\frac{\left(e_{i}-e_{j}\right)\left(e_{i}-e_{j}\right)^{T}}{2}: \quad a_{i j}+a_{j i}>0, i \neq j\right\} .
$$

Lemma 5.2: Let $M_{k} \in \mathbb{M}, k=1, \ldots, N$ be $N \geq 1$ matrices in $\mathbb{M}$. Then we have $\left(\bigcup_{i=1}^{N} \mathcal{G}_{M_{i}}\right) \subseteq \mathcal{G}_{M_{N} \ldots M_{1}}$.

\begin{tabular}{|c|c|c|}
\hline & $\begin{array}{c}\text { Perfectly Dependent } \\
\text { Communication }\end{array}$ & $\begin{array}{c}\text { Independent } \\
\text { Communication }\end{array}$ \\
\hline $\begin{array}{c}\text { Critical Underlying } \\
\text { Connectivity }\end{array}$ & Weak Connectivity & Double Connectivity \\
\hline $\begin{array}{c}\text { Consensus Limit }= \\
\text { Initial Average }\end{array}$ & Almost Surely & In Expectation \\
\hline Average Preserved & $\begin{array}{c}\text { Almost Forever } \\
\text { (for all } \mathbf{n} \text { ) }\end{array}$ & $\begin{array}{c}\text { Almost Never } \\
\text { (for odd } \mathbf{n} \text { ) }\end{array}$ \\
\hline
\end{tabular}

Fig. 1. Summary of the properties of the random gossip algorithms considered in the paper. Perfectly dependent and independent communication gives drastically different behavior.

Moreover, all nonzero entries of $M_{N} \ldots M_{1}$ have lower bound $2^{-N}$.

Denoting $d_{*}=\max \left\{\operatorname{Diag}\left(\mathcal{G}_{\mathrm{A}}\right), \operatorname{Diag}\left(\mathcal{G}_{\mathrm{A}^{\mathrm{T}}}\right)\right\}$, where $\operatorname{Diag}\left(\mathcal{G}_{\mathrm{A}}\right)$ and $\operatorname{Diag}\left(\mathcal{G}_{\mathrm{AT}}\right)$ represent the diameter of the induced graph of $A$ and $A^{T}$, respectively. We have the following lemma.

Lemma 5.3: Suppose the double connectivity assumption, A4 holds. Let $M_{1}, \ldots, M_{2 d_{*}-1}$ be $2 d_{*}-1$ products of some finite matrices in M. If either $\mathcal{G}_{A} \subseteq \mathcal{G}_{M_{k}}$ or $\mathcal{G}_{A^{T}} \subseteq \mathcal{G}_{M_{k}}$ holds for any $k=1, \ldots, 2 d_{*}-1$, then $M_{2 d_{*}-1} \ldots M_{1}$ is a scrambling matrix.

We further denote $\mathbb{M}^{*}=\mathbb{M}_{1}^{*} \cup \mathbb{M}_{2}^{*}$ with $\mathbb{M}_{1}^{*}=\{I-$ $\left.\frac{e_{i}\left(e_{i}-e_{j}\right)^{T}}{2}: i, j=1, \ldots, n, i \neq j\right\}$ and $\mathbb{M}_{2}^{*}=\{I-$ $\left.\frac{\left(e_{i}-e_{j}\right)\left(e_{i}-e_{j}\right)^{T}}{2}: i, j=1, \ldots, n, i \neq j\right\}$.

Lemma 5.4: Suppose $n$ is an odd number. Take matrices $M_{\tau} \in \mathbb{M}_{2}^{*}, \tau=1, \ldots, k, k \geq 1$ arbitrarily. Then we have $\delta\left(M_{k} \cdots M_{1}\right)>0$, where $\delta(\cdot)$ is defined in (1).

The proofs of Theorems 5.1, 5.2, 5.3 and 5.4 are based on Lemmas 5.1, 5.2, 5.3 and 5.4. Again we refer to [44] for technical details.

\section{CONCLUSIONS}

This paper presented new results on the role of unreliable node communication in the convergence of randomized gossip algorithms. The model for the random node pair selection process is defined by a stochastic matrix which characterizes the interactions among the nodes in the network. A pair of nodes meets at a random instance, and two Bernoulli communication links are then established between the nodes. Communication on each link succeeds with a time-dependent probability. We presented a series of necessary and sufficient conditions on the success probability sequence to ensure a.s. consensus or $\epsilon$-consensus under perfectly dependent and independent communication processes, respectively. The results showed that the communication symmetry is critical for the convergence.

The results are summarized in the following table. We notice the following characteristics:

- In terms of consensus convergence of the randomized gossip algorithm, A1 (Weak Connectivity) is critical for perfectly dependent communication, as is A4 (Double Connectivity) for independent communication. 
- For perfectly dependent communication, the consensus limit equals the initial average with probability one. While for independent communication, only the expected value of the consensus limit equals the initial average for the special case $P_{k}^{+}=P_{k}^{-}$.

- Average is preserved almost forever (with probability one for all initial conditions) with perfectly dependent communication, and it is preserved almost never (with probability zero for almost all initial conditions) with independent communication if the number of nodes is odd.

The results illustrate that convergence behavior of distributed algorithms may heavily depend on the probabilistic dependence properties in the information flow.

\section{REFERENCES}

[1] D. P. Bertsekas and J. N. Tsitsiklis. Introduction to Probability. Athena Scientific, Massachusetts, 2002.

[2] G. Latouche, V. Ramaswami. Introduction to Matrix Analytic Methods in Stochastic Modeling. 1st edition, ASA SIAM, 1999.

[3] C. Godsil and G. Royle. Algebraic Graph Theory. New York: SpringerVerlag, 2001.

[4] A. Benveniste, M. Métivier and P. Priouret. Adaptive Algorithms and Stochastic Approximations. Springer-Verlag: Berlin, 1990.

[5] B. Bollobás. Random Graphs. Cambridge University Press, second edition, 2001.

[6] P. Gupta and P. R. Kumar, "Critical power for asymptotic connectivity in wireless networks," Stochastic Analysis, Control, Optimization and Applications: A Volume in Honor of W.H. Fleming, 547-566, 1998

[7] P. Erdös and A. Rényi, "On the evolution of random graphs," $P u b$ lications of the Mathematical Institute of the Hungarian Academy of Sciences, 17-61, 1960.

[8] C. Berge and A. Ghouila-Houri. Programming, Games, and Transportation Networks, John Wiley and Sons, New York, 1965.

[9] D. Kempe, A. Dobra, and J. Gehrke, "Gossip-based computation of aggregate information, in Proc. Conf. Foundations of Computer Science, pp. 482-491, 2003.

[10] R. Karp, C. Schindelhauer, S. Shenker, and B. Vcking, "Randomized rumor spreading, in Proc. Symp. Foundations of Computer Science, pp. 564-574, 2000.

[11] S. Boyd, P. Diaconis and L. Xiao, "Fastest mixing markov chain on a graph," SIAM Review, Vol. 46, No. 4, pp. 667-689, 2004.

[12] S. Boyd, A. Ghosh, B. Prabhakar and D. Shah, "Randomized gossip algorithms," IEEE Trans. Information Theory, vol. 52, no. 6, pp. 25082530, 2006.

[13] D. Mosk-Aoyama and D. Shah, "Fast distributed algorithms for computing separable functions," IEEE Transactions on Information Theory, vol.55, no.7, pp. 2997-3007, 2008

[14] D. Shah, "Gossip Algorithms," Foundations and Trends in Networking, Vol. 3, No. 1, pp. 1-125, 2008.

[15] J. Wolfowitz, "Products of indecomposable, aperiodic, stochastic matrices," Proc. Amer. Math. Soc., vol. 15, pp. 733-736, 1963.

[16] J. Hajnal, "Weak ergodicity in non-homogeneous markov chains," Proc. Cambridge Philos. Soc., no. 54, pp. 233-246, 1958.

[17] M. H. DeGroot, "Reaching a consensus," Journal of the American Statistical Association, vol. 69, no. 345, pp. 118-121, 1974.

[18] S. Muthukrishnan, B. Ghosh, and M. Schultz, "First and second order diffusive methods for rapid, coarse, distributed load balancing," Theory of Computing Systems, vol. 31, pp. 331-354, 1998.

[19] R. Diekmann, A. Frommer, and B. Monien, "Efficient schemes for nearest neighbor load balancing," Parallel Computing, vol. 25, pp. 789812, 1999.

[20] S. Martinez, J. Cortés, and F. Bullo, "Motion coordination with distributed information," IEEE Control Systems Magazine, vol. 27, no. 4, pp. 75-88, 2007.

[21] J. Tsitsiklis, D. Bertsekas, and M. Athans, "Distributed asynchronous deterministic and stochastic gradient optimization algorithms," IEEE Trans. Autom. Control, vol. 31, pp. 803-812, 1986.
[22] A. Jadbabaie, J. Lin, and A. S. Morse, "Coordination of groups of mobile autonomous agents using nearest neighbor rules, IEEE Trans. Autom.Control, vol. 48, no. 6, pp. 988-1001, 2003.

[23] R. Olfati-Saber and R. Murray, "Consensus problems in the networks of agents with switching topology and time dealys," IEEE Trans. Autom. Control, vol. 49, no. 9, pp. 1520-1533, 2004.

[24] J. Fax and R. Murray, "Information flow and cooperative control of vehicle formations," IEEE Trans. Autom. Control, vol. 49, no. 9, pp. 1465-1476, 2004.

[25] M. Cao, A. S. Morse and B. D. O. Anderson, "Reaching a consensus in a dynamically changing environment: a graphical approach," SIAM J. Control Optim., vol. 47, no. 2, 575-600, 2008.

[26] M. Cao, A. S. Morse and B. D. O. Anderson, "Reaching a consensus in a dynamically changing environment: convergence rates, measurement delays, and asynchronous events," SIAM J. Control Optim., vol. 47, no. 2, 601-623, 2008.

[27] M. Cao, A. S. Morse and B. D. O. Anderson, "Agreeing asynchronously," IEEE Trans. Autom. Control, vol. 53, no. 8, 1826-1838, 2008.

[28] W. Ren and R. Beard, "Consensus seeking in multi-agent systems under dynamically changing interaction topologies," IEEE Trans. Autom. Control, vol. 50, no. 5, pp. 655-661, 2005.

[29] L. Moreau, "Stability of multi-agent systems with time-dependent communication links," IEEE Trans. Autom. Control, vol. 50, pp. 169$182,2005$.

[30] Y. Hatano and M. Mesbahi, "Agreement over random networks," IEEE Trans. on Autom. Control, vol. 50, no. 11, pp. 1867-1872, 2005.

[31] C. W. Wu, "Synchronization and convergence of linear dynamics in random directed networks," IEEE Trans. Autom. Control, vol. 51, no. 7, pp. 1207-1210, 2006.

[32] A. Tahbaz-Salehi and A. Jadbabaie, "A necessary and sufficient condition for consensus over random networks," IEEE Trans. on Autom. Control, VOL. 53, NO. 3, pp. 791-795, 2008.

[33] F. Fagnani and S. Zampieri, "Randomized consensus algorithms over large scale networks," IEEE J. on Selected Areas of Communications, vol. 26, no.4, pp. 634-649, 2008.

[34] F. Fagnani and S. Zampieri, "Average consensus with packet drop communication," SIAM J. Control Optim., vol. 48, no. 1, pp. 102-133, 2009.

[35] S. Patterson, B. Bamieh and A. El Abbadi, "Convergence rates of distributed average consensus with stochastic link failures," IEEE Trans. Autom. Control, vol. 55, no. 4, pp. 880-892, 2010.

[36] C. C. Moallemi and B. Van Roy, "Consensus propagation," IEEE Trans. Information Theory, vol. 52, no. 11, pp. 4753-4766, 2006.

[37] T. C. Aysal and K. E. Barner, "Convergence of consensus models with stochastic disturbances," IEEE Trans. Information Theory, vol. 56, no. 8, pp. 4101-4113, 2010.

[38] S. Kar and J. M. F. Moura, "Distributed consensus algorithms in sensor networks: quantized data and random link failures," IEEE Trans. Signal Processing, Vol. 58:3, pp. 1383-1400, 2010.

[39] U. A. Khan, S. Kar, and J. M. F. Moura, "Distributed sensor localization in random environments using minimal number of anchor nodes, IEEE Trans. Signal Processing, 57: 5, pp. 2000-2016, 2009.

[40] A. Nedić, A. Olshevsky, A. Ozdaglar, and J. N. Tsitsiklis, "On distributed averaging algorithms and qantization effects," IEEE Trans. Autom. Control, vol. 54, no. 11, pp. 2506-2517, 2009.

[41] D. Acemoglu, A. Ozdaglar and A. ParandehGheibi, "Spread of (Mis)information in social networks," Games and Economic Behavior, vol. 70, no. 2, pp. 194-227, 2010.

[42] D. Acemoglu, G. Como, F. Fagnani, A. Ozdaglar, "Opinion fluctuations and persistent disagreement in social networks," in IEEE Conference on Decision and Control, Orlando, 2011.

[43] B. Touri and A. Nedić, "On ergodicity, infinite flow and consensus in random models," IEEE Transactions on Automatic Control, vol 56, pp. 1593-1605, 2011.

[44] G. Shi, M. Johansson and K. H. Johansson, "Randomized gossip algorithm with unreliable communication," preprint at arxiv.org, avaiable from http://arxiv.org/pdf/1203.6028v1.pdf 